\title{
Anisotropic admixture in color-superconducting quark matter
}

\author{
Michael Buballa, ${ }^{1,2}$ Jiří Hošek, ${ }^{3}$ and Micaela Oertel ${ }^{4}$ \\ ${ }^{1}$ Institut für Kernphysik, Schlossgartenstr. 9, D-64289 Darmstadt, Germany \\ ${ }^{2}$ Gesellschaft für Schwerionenforschung, Planckstr. 1, D-64291 Darmstadt, Germany \\ ${ }^{3}$ Dept. Theoretical Physics, Nuclear Physics Institute, 25068 Řež (Prague), Czech Republic \\ ${ }^{4}$ IPN-Lyon, 43 Bd du 11 Novembre 1918, 69622 Villeurbanne Cédex, France
}

(Dated: April 2, 2003)

\begin{abstract}
The analysis of color-superconducting two-flavor deconfined quark matter at moderate densities is extended to include a particular spin-1 Cooper pairing of those quarks which do not participate in the standard spin-0 diquark condensate. (i) The relativistic spin- 1 gap $\Delta^{\prime}$ implies spontaneous breakdown of rotation invariance manifested in the form of the quasi-fermion dispersion law. (ii) The critical temperature of the anisotropic component is approximately given by the relation $T_{c}^{\prime} \simeq \Delta^{\prime}(T=0) / 3$. (iii) For massless fermions the gas of anisotropic Bogolyubov-Valatin quasiquarks becomes effectively gapless and two-dimensional. Consequently, its specific heat depends quadratically on temperature. (iv) All collective Nambu-Goldstone excitations of the anisotropic phase have a linear dispersion law and the whole system remains a superfluid. (v) The system exhibits an electromagnetic Meissner effect.
\end{abstract}

PACS numbers: 12.39.Ki, 12.38.Aw,11.30.Qc

Recent investigations suggest that the phase structure of QCD is very rich 1, 2]. At low temperatures and high densities strongly interacting matter is expected to be a color superconductor 3]. At asymptotically high densities, where the QCD coupling constant becomes small, this can be analyzed starting from first principles [4, 5], whereas at more moderate densities, present (presumably) in the interiors of neutron stars, these methods are no longer justified. In this region the low-energy dynamics of deconfined quark matter is often studied employing effective Lagrangians $\mathcal{L}_{\text {eff }}$ which contain local or non-local four-fermion interactions, most importantly interactions derived from instantons or on a more phenomenological basis [6, 7, 8]. The non-confining gluon $S U(3)_{c}$ gauge fields are then treated as weak external perturbations, and neglected in lowest approximation.

In this letter we consider the case of two flavors which is most likely relevant at chemical potentials just above the deconfinement phase transition. On physical grounds it is then natural to assume that $\mathcal{L}_{\text {eff }}$ favors the spontaneous formation of spin-0 isospin singlet Cooper pair condensates [1, 9] $\delta=\left\langle\psi^{T} C \gamma_{5} \tau_{2} \lambda_{2} \psi\right\rangle$, where $\psi$ is a quark field, $C$ the matrix of charge conjugation, $\tau_{2}$ a Pauli matrix which acts in flavor space, and $\lambda_{2}$ a GellMann matrix which acts in color space. Due to the latter $S U(3)_{c}$ is broken down to $S U(2)_{c}$. This has the following consequences for the physical excitations of the system:

(i) Corresponding to the mixing of the colors 1 and 2 there are two Bogolyubov-Valatin quasiquarks for each flavor with the dispersion law $E_{1}^{ \pm}(\vec{p})=E_{2}^{ \pm}(\vec{p}) \equiv$ $E^{ \pm}(\vec{p})=\sqrt{\left(\epsilon_{p} \pm \mu\right)^{2}+|\Delta|^{2}}$. The energy gap $\Delta$ is the solution of a selfconsistent gap equation and is found to be typically of the order $\sim 100 \mathrm{MeV}$ in model calculations [6, 7], [8]. $\epsilon_{p}=\sqrt{\vec{p}^{2}+M^{2}}$, where $M$ is an effective Dirac mass, related to the chiral condensate $\langle\bar{\psi} \psi\rangle$ via a selfcon- sistency equation 8 . For each flavor there is an unpaired quark of color 3 with the dispersion law $\epsilon_{3}^{ \pm}(\vec{p})=\epsilon_{p} \pm \mu$.

(ii) Because of the spontaneous breaking of $S U(3)_{c}$ down to $S U(2)_{c}$ five of the eight gluons receive a mass (Meissner effect), whereas three remain massless [10]. Since no global symmetry is spontaneously broken there are no massless Goldstone bosons.

(iii) The condensate $\delta$ is invariant under a local $U(1)$ transformation generated by $\tilde{Q}=Q-\frac{1}{2 \sqrt{3}} \lambda_{8}$, where $Q$ is the electromagnetic charge operator and $\lambda_{8}$ a Gell-Mann matrix in color space. As long as this symmetry is not broken by other condensates, there is a "new" photon (a linear combination of the "normal" photon and the eighth gluon) which remains massless. This means, there is no electromagnetic Meissner effect.

According to Cooper's theorem any attractive interaction leads to an instability at the Fermi surface. It is therefore rather unlikely, that the Fermi sea of color-3 quarks stays intact. As only quarks of a single color are involved, the pairing must take place in a channel which is symmetric in color. Assuming $s$-wave condensation in an isospin-singlet channel, a possible candidate is a spin1 condensate [6]. Although the size of the corresponding gap was estimated to be much smaller than $\Delta[\underline{6}]$, its existence can have important astrophysical consequences. For example, if all quarks are gapped, the specific heat of a potential quark core of a neutron star (and hence the cooling of the star) is goverened by the size of the smallest gap [1]. The same is true for other transport properties, like neutrino emissivity or viscosity.

This letter is devoted to a quantitative analysis of this possibility. To this end we consider the condensate

$$
\delta^{\prime}=\left\langle\psi^{T} C \sigma^{03} \tau_{2} \hat{P}_{3}^{(c)} \psi\right\rangle,
$$

where $\sigma^{\mu \nu}=i / 2\left[\gamma^{\mu}, \gamma^{\nu}\right]$ and $\hat{P}_{3}^{(c)}=1 / 3-1 / \sqrt{3} \lambda_{8}$ is 
the projector on color $3 . \delta^{\prime}$ is a ground-state expectation value of a complex vector order parameter $\phi^{0 n} \equiv \phi_{n}$ describing spin 1 and breaking spontaneously the rotational invariance of the system. There are well-known examples for spin-1 pairing in condensed matter physics, e.g., superfluid ${ }^{3} \mathrm{He}$, where some phases are also anisotropic [11]. In relativistic systems this is certainly not a very frequent phenomenon. It is possible only at finite chemical potential, which itself breaks Lorentz invariance explicitly. (Relativistic Cooper pairing into spin-1 with nonzero angular momentum was considered elsewhere, e.g., [9, 12].) Another example of non-isotropic quark pairing are crystalline color superconductors 13. The role of spin- 1 condensates as an alternative to crystals in single color or single flavor systems has also been discussed in Ref. 14].

Since rotational invariance is a global symmetry of the primary Lagrangian, an arbitrary small gap of the anisotropic phase implies specific gapless collective excitations with given Landau critical velocity crucial for the superfluid behavior of the system. We will briefly discuss this at the end of this Letter. It is also interesting to note that $\delta^{\prime}$ is not neutral with respect to the "rotated" electric charge $\tilde{Q}$ and there is no generalized electric charge for which both, $\delta$ and $\delta^{\prime}$, are neutral. This means, if both, $\delta$ and $\delta^{\prime}$, were present in a neutron star, there would be an electromagnetic Meissner effect, which would strongly influence the magnetic field. Recently, similar effects have been discussed in Ref. 15]. The detailed evaluation of the Meissner masses for our case remains to be done.

For the quantitative analysis we have to specify the interaction. Guided by the structure of instanton-induced interactions (see, e.g., 7]) we consider a quark-antiquark term

$$
\mathcal{L}_{q \bar{q}}=G\left\{(\bar{\psi} \psi)^{2}-(\bar{\psi} \vec{\tau} \psi)^{2}-\left(\bar{\psi} i \gamma_{5} \psi\right)^{2}+\left(\bar{\psi} i \gamma_{5} \vec{\tau} \psi\right)^{2}\right\}
$$

and a quark-quark term

$$
\begin{aligned}
\mathcal{L}_{q q}= & -H_{s} \sum_{\mathcal{O}=\gamma_{5}, 1}\left(\bar{\psi} \mathcal{O} C \tau_{2} \lambda_{A} \bar{\psi}^{T}\right)\left(\psi^{T} C \mathcal{O} \tau_{2} \lambda_{A} \psi^{T}\right) \\
& -H_{t}\left(\bar{\psi} \sigma^{\mu \nu} C \tau_{2} \lambda_{S} \bar{\psi}^{T}\right)\left(\psi^{T} C \sigma_{\mu \nu} \tau_{2} \lambda_{S} \psi^{T}\right)
\end{aligned}
$$

where $\lambda_{A}$ and $\lambda_{S}$ are the antisymmetric and symmetric color generators, respectively. For instanton induced interactions the coupling constants fulfill the relation $G: H_{s}: H_{t}=1: \frac{3}{4}: \frac{3}{16}$, but for the moment we will treat them as arbitrary parameters. As long as they stay positive, the interaction is attractive in the channels giving rise to the diquark condensates $\delta$ and $\delta^{\prime}$ as well as to the chiral condensate $\langle\bar{\psi} \psi\rangle$. It is straight forward to calculate the mean-field thermodynamic potential $\Omega(T, \mu)$ in the presence of these condensates:

$$
\begin{aligned}
\Omega(T, \mu)= & -4 \sum_{i=1}^{3} \sum_{+-} \int \frac{d^{3} p}{(2 \pi)^{3}}\left[\frac{E_{i}^{ \pm}}{2}+T \ln \left(1+e^{-E_{i}^{ \pm} / T}\right)\right] \\
& +\frac{1}{4 G}(M-m)^{2}+\frac{1}{4 H_{s}}|\Delta|^{2}+\frac{1}{16 H_{t}}\left|\Delta^{\prime}\right|^{2}, \quad(4)
\end{aligned}
$$

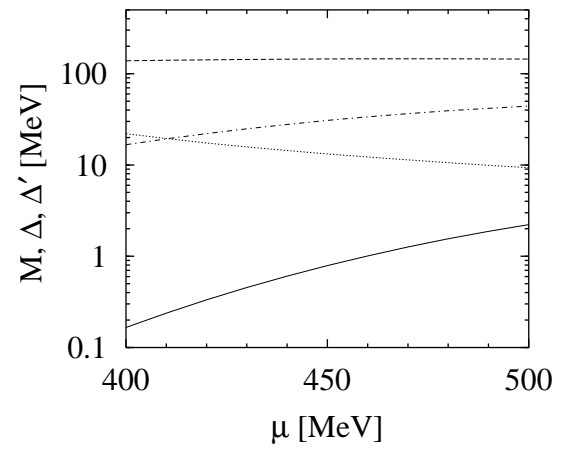

FIG. 1: $M$ (dotted), $\Delta$ (dashed), and $\Delta^{\prime}$ (solid) at $T=0$ as functions of the quark chemical potential $\mu$ using parameter set 1 (see text). The dashed-dotted line indicates the result for $\Delta^{\prime}$ taking parameter set 2 .

where $m$ is the bare quark mass, $M=m-2 G\langle\bar{\psi} \psi\rangle$, $\Delta=-2 H_{s} \delta$, and $\Delta^{\prime}=4 H_{t} \delta^{\prime}$. The dispersion law for quarks of color 3 reads

$$
E_{3}^{\mp}(\vec{p})=\sqrt{\left(\sqrt{M_{e f f}^{2}+\vec{p}^{2}} \mp \mu_{e f f}\right)^{2}+\left|\Delta_{e f f}^{\prime}\right|^{2}},
$$

where $\mu_{\text {eff }}^{2}=\mu^{2}+\left|\Delta^{\prime}\right|^{2} \sin ^{2} \theta, M_{\text {eff }}=M \mu / \mu_{\text {eff }}$, and $\left|\Delta_{\text {eff }}^{\prime}\right|^{2}=\left|\Delta^{\prime}\right|^{2}\left(\cos ^{2} \theta+M^{2} / \mu_{\text {eff }}^{2} \sin ^{2} \theta\right)$. Here $\cos \theta=$ $p_{3} /|\vec{p}|$. Thus, as expected, for $\Delta^{\prime} \neq 0, E_{3}^{ \pm}(\vec{p})$ is an anisotropic function of $\vec{p}$, clearly exhibiting the spontaneous breakdown of rotational invariance. For $M=0$, the gap $\Delta_{\text {eff }}^{\prime}$ vanishes at $\theta=\pi / 2$. In general its minimal value is given by $\Delta_{0}^{\prime}=M\left|\Delta^{\prime}\right| / \sqrt{\mu^{2}+\left|\Delta^{\prime}\right|^{2}}$. Expanding $E_{3}^{-}$around its minimum the low-lying quasiparticle spectrum takes the form

$$
E_{3}^{-}\left(p_{\perp}, p_{3}\right) \approx \sqrt{\Delta_{0}^{\prime 2}+v_{\perp}^{2}\left(p_{\perp}-p_{0}\right)^{2}+v_{3}^{2} p_{3}^{2}},
$$

where $v_{\perp}=\left(1-\left(\mu \Delta^{\prime 2}{ }_{0}^{2} /\left(M \Delta^{\prime 2}\right)\right)^{2}\right)^{1 / 2}, v_{3}=\frac{\Delta_{0}^{\prime}}{M}, p_{0}=$ $\frac{v_{\perp}}{v_{3}}\left|\Delta^{\prime}\right|$, and $p_{\perp}^{2}=p_{1}^{2}+p_{2}^{2}$. This leads to a density of states linear in energy:

$$
N(E)=\frac{1}{2 \pi} \frac{\mu^{2}+\left|\Delta^{\prime}\right|^{2}}{\left|\Delta^{\prime}\right|} E \theta\left(E-\Delta_{0}^{\prime}\right) .
$$

The actual values for $\Delta, \Delta^{\prime}$ and $M$ follow from the condition that the stable solutions correspond to the absolute minimum of $\Omega$ with respect to these quantities. Imposing $\partial \Omega / \partial \Delta^{\prime *}=0$ leads to the following gap equation for $\Delta^{\prime}$ :

$$
\Delta^{\prime}=16 H_{t} \Delta^{\prime} \sum_{+-} \int \frac{d^{3} p}{(2 \pi)^{3}}\left(1 \pm \frac{\vec{p}_{\perp}^{2}}{s}\right) \frac{1}{E_{3}^{ \pm}} \tanh \frac{E_{3}^{ \pm}}{2 T},
$$

where $s=\mu_{\text {eff }}\left(\vec{p}^{2}+M_{\text {eff }}^{2}\right)^{1 / 2}$. Similarly one can derive gap equations for $\Delta$ and $M$ by the requirements $\partial \Omega / \partial \Delta^{*}=0$ and $\partial \Omega / \partial M=0$, respectively. Together with Eq. (8), they form a set of three coupled equations, 


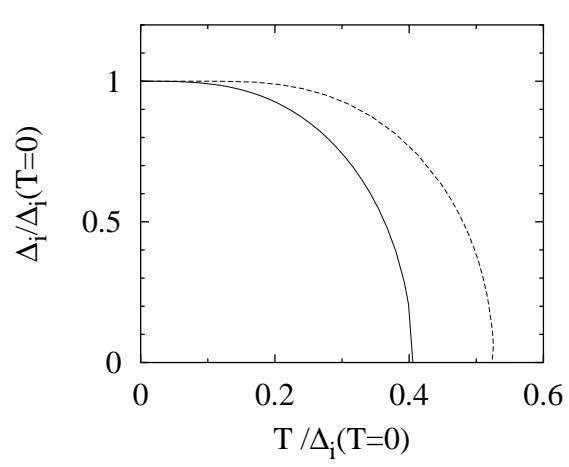

FIG. 2: $\Delta_{i} / \Delta_{i}(T=0)$ as function of $T / \Delta_{i}(T=0)$. Dashed: $\Delta_{i}=\Delta$. Solid: $\Delta_{i}=\Delta^{\prime}$. The calculations have been performed at $\mu=450 \mathrm{MeV}$ for parameter set 1 .

which have to be solved simultaneously. However, the equations for $\Delta$ and $\Delta^{\prime}$ are not directly coupled, but only through their dependence on $M$.

In our numerical calculations we use a sharp 3momentum cutoff $\Lambda$ to regularize the integrals. We then have five parameters: $m, \Lambda, G, H_{s}$, and $H_{t}$. We choose $m=5 \mathrm{MeV}, \Lambda=600 \mathrm{MeV}$, and $G \Lambda^{2}=2.4$ - leading to reasonable vacuum properties, $M=393 \mathrm{MeV}$ and $\langle\bar{u} u\rangle=(-244 \mathrm{MeV})^{3}-$, and the instanton relation to fix $H_{s}$ and $H_{t}$ ("parameter set 1 "). The resulting values of $M, \Delta$, and $\Delta^{\prime}$ as functions of $\mu$ at $T=0$ are displayed in Fig. [1 The chemical potentials correspond to baryon densities of about 4 - 7 times nuclear matter density. In agreement with earlier expectations [6] $\Delta^{\prime}$ is small compared with $\Delta$. However, its value rises strongly with $\mu$. Being a solution of a selfconsistency problem, $\Delta^{\prime}$ is also extremely sensitive to the coupling constant $H_{t}$. If we double the value of $H_{t}$ ("parameter set 2"), we arrive at the dashed-dotted line for $\Delta^{\prime}$, which is then comparable to $\Delta$. As a consequence of the factor $\left(1-\vec{p}_{\perp}^{2} / s\right)$ in the gap equation (8), $\Delta^{\prime}$ is very sensitive to value and the form of the cutoff.

With increasing temperature both condensates, $\delta$ and $\delta^{\prime}$, are reduced and eventually vanish in second-order phase transitions at critical temperatures $T_{c}$ and $T_{c}^{\prime}$, respectively. It has been shown [5] that $T_{c}$ is approximately given by the well-known BCS relation $T_{c} \simeq 0.57 \Delta(T=0)$. In order to derive a similar relation for $T_{c}^{\prime}$ we inspect the gap equation (8) at $T=0$ and in the limit $T \rightarrow T_{c}^{\prime}$. Neglecting $M$ (since $M \ll \mu$ this is valid up to higher orders in $M^{2} / \mu^{2}$ ) and antiparticle contributions one gets

$$
\begin{aligned}
\int \frac{d^{3} p}{(2 \pi)^{3}}\left\{\left[\left(1-\frac{\vec{p}_{\perp}^{2}}{s}\right) \frac{1}{E_{3}^{-}(\vec{p})}\right]_{\Delta^{\prime}(T=0)}\right. \\
\left.-\left(1-\frac{\vec{p}_{\perp}^{2}}{\mu|\vec{p}|}\right) \frac{1}{|\mu-| \vec{p}||} \tanh \frac{|\mu-| \vec{p}||}{2 T_{c}^{\prime}}\right\} \approx 0 .
\end{aligned}
$$

Since the integrand is strongly peaked near the Fermi surface, the $|\vec{p}|$-integrand must approximately vanish at $|\vec{p}|=\mu$, after the angular integration has been performed.

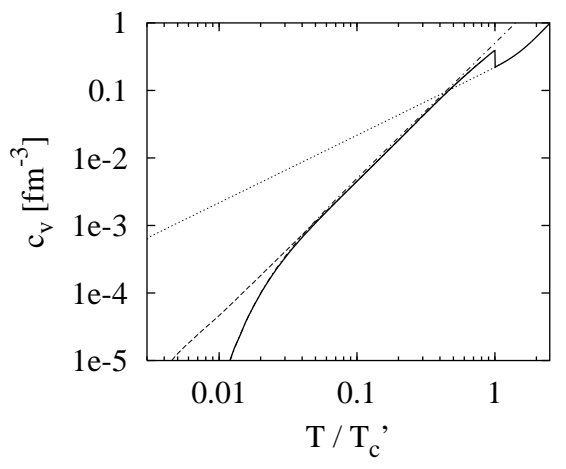

FIG. 3: Specific heat for parameter set 2 at $\mu=450 \mathrm{MeV}$ as function of $T / T_{c}^{\prime}$. Solid: full calculation, dashed: result for $M=0$, dotted: without spin-1 condensate. The dasheddotted line indicates the result of Eq. (10).

From this condition one finds to lowest order in $\Delta^{\prime} / \mu$ : $T_{c}^{\prime} / \Delta^{\prime}(T=0) \approx 1 / 3$. The analogous steps would lead to $T_{c} / \Delta(T=0) \approx 1 / 2$ instead of the textbook value of 0.57 . This gives a rough idea about the quality of the approximation. Note that there are other examples of diquark condensates, where $T_{c} \neq 0.57 \Delta(T=0)[16$. This is also the case for crystalline superconductors [17].

Numerical results for $\Delta(T)$ and $\Delta^{\prime}(T)$ are shown in Fig. 2. The quantities have been rescaled in order to facilitate a comparison with the above relations for $T_{c}$ and $T_{c}^{\prime}$. Our results are in reasonable agreement with our estimates. These findings turn out to be insensitive to the actual choice of parameters.

The specific heat is given by $c_{v}=-T \partial^{2} \Omega / \partial T^{2}$ [24]. For $T \ll T_{c}$ it is completely dominated by quarks of color 3 , since the contribution of the first two colors is suppressed by a factor $\mathrm{e}^{-\Delta / \mathrm{T}}$. Neglecting the $T$-dependence of $M$ and $\Delta^{\prime}$, and employing Eq. (7), one finds

$$
c_{v} \approx \frac{12}{\pi} \frac{\mu^{2}+\left|\Delta^{\prime}\right|^{2}}{\left|\Delta^{\prime}\right|} T^{2} e^{-\frac{\Delta_{0}^{\prime}}{T}} \sum_{n=0}^{3} \frac{1}{n !}\left(\frac{\Delta_{0}^{\prime}}{T}\right)^{n},
$$

which should be valid for $T \ll T_{c}^{\prime}$. In this regime $c_{v}$ depends quadratically on $T$ for $T \gtrsim \Delta_{0}^{\prime}$, and is exponentially suppressed at lower temperatures. To test this relation we evaluate $c_{v}(T)$ explicitly using Eq. (4). The results for fixed $\mu=450 \mathrm{MeV}$ are displayed in Fig. 3 For numerical convenience we choose parameter set 2 , leading to a relatively large $\Delta^{\prime}(T=0)=30.8 \mathrm{MeV}$. The critical temperature is $T_{c}^{\prime} \simeq 0.40 \Delta^{\prime}(T=0)$. For the energy gap we find $\Delta_{0}^{\prime}=0.074 T_{c}^{\prime}$. It turns out that Eq. (10), evaluated with constant values of $\Delta^{\prime}$ and $M$, (dashed-dotted) is in almost perfect agreement with the numerical result (solid) up to $T \approx T_{c}^{\prime} / 2$. The phase transition, causing the discontinuity of $c_{v}$ at $T=T_{c}^{\prime}$, is of course outside the range of validity of Eq. (10). We also display $c_{v}$ for $M=0$ (dashed). Since $\Delta_{0}^{\prime}$ vanishes in this case there is no exponential suppression, and $c_{v}$ is proportional to $T^{2}$ down to arbitrarily low $T$. However, even when $M$ is included, the exponential suppression is partially cancelled 
by the sum on the r.h.s. of Eq. (10). For comparison we also show $c_{v}$ for a system with $\Delta^{\prime}=0$, which exhibits a linear $T$ dependence at low temperatures (dotted).

Our results show that, even though the magnitude of the gap parameter $\Delta^{\prime}$ is strongly model dependent its relations to the critical temperature and the specific heat are quite robust. Thus, if we had empirical data, e.g., for the specific heat of dense quark matter, they could be used to extract information about the existence and the size of $\Delta^{\prime}$. In this context neutron stars and their cooling properties are the natural candidates to look at. In Ref. 1] it was suggested that the exponential suppression of $c_{v}$ related to the potential pairing of quarks of color 3 might have observable consequences for the neutrino emission of a neutron star. This argument has to be somewhat refined since, as seen above, $c_{v}(T)$ first behaves as $T^{2}$ and the exponential suppression sets in only at $T<\Delta_{0}^{\prime}$. The relevance of $c_{v}$ and the possible effect of diquark condensates on neutron star cooling was also discussed in Ref. 19. On the other hand it has recently been argued 20], that the constraints imposed by charge and color neutrality might completely prohibit the existence of two-flavor color-superconducting matter in neutron stars.

Because of the spontaneously broken $U(1) \times O(3)$ symmetry in Eq. (11), for $\Delta^{\prime} \neq 0$ there should be collective Nambu-Goldstone excitations in the spectrum. However, due to the Lorentz non-invariance of the system there can be subtleties [21, 22, 23]. The NG spectrum can be analyzed within an underlying effective Higgs potential

$$
V(\phi)=-a^{2} \phi_{n}^{\dagger} \phi_{n}+\frac{1}{2} \lambda_{1}\left(\phi_{n}^{\dagger} \phi_{n}\right)^{2}+\frac{1}{2} \lambda_{2} \phi_{n}^{\dagger} \phi_{n}^{\dagger} \phi_{m} \phi_{m},
$$

for the complex order parameter $\phi_{n}$ [22], with $\lambda_{1}+\lambda_{2}>0$ for stability. For $\lambda_{2}<0$ the ground state is characterized by $\phi_{\text {vac }}^{(1)}=\left(\frac{a^{2}}{\lambda_{1}}\right)^{1 / 2}(0,0,1)$ which corresponds to our ansatz Eq. (11) for the BCS-type diquark condensate $\delta^{\prime}$. This solution has the property $\langle\vec{S}\rangle^{2}=\left(\phi_{v a c}^{(1) \dagger} \vec{S} \phi_{v a c}^{(1)}\right)^{2}=0$. The spectrum of small oscillations above $\phi_{v a c}^{(1)}$ consists of $1+2 \mathrm{NG}$ bosons, all with linear dispersion law: one zero-sound phonon and two spin waves 22]. Implying a finite Landau critical velocity, this fact is crucial for a macroscopic superfluid behavior of the system [23].

Note that for $\lambda_{2}>0$ there is a different solution $\phi_{\text {vac }}^{(2)}=\left(\frac{a^{2}}{2\left(\lambda_{1}+\lambda_{2}\right)}\right)^{1 / 2}(1, i, 0)$ with $\langle\vec{S}\rangle^{2}=1$. In this case the NG spectrum above $\phi_{v a c}^{(2)}$ consists of one phonon with linear dispersion law and one spin wave whose energy tends to zero with momentum squared [22]. The quasiquark dispersion law corresponding to $\phi_{v a c}^{(2)}$ has recently been discussed in Ref. [14]. A detailed analysis of the transport properties implied by this type of pairing seems of particular interest for the phenomenology of neutron stars since the dispersion law allows for gapless excitations even for massive quarks [14].

We thank D. Blaschke, D. Litim, K. Rajagopal, I.A.
Shovkovy, and E.V. Shuryak for useful discussions. J.H. thanks J. Wambach and IKP TU Darmstadt for generous hospitality and support. We acknowledge financial support by ECT* during its 2001 collaboration meeting on color superconductivity. This work was supported in part by grant GACR 202/02/0847. M.O. acknowledges support from the Alexander von Humboldt-foundation.

[1] K. Rajagopal and F. Wilczek, hep-ph/0011333 and references therein.

[2] M. Alford, Ann. Rev. Nucl. Part. Sci. 51, 131 (2001).

[3] J.C. Collins and M.J. Perry, Phys. Rev. Lett. 34, 1353 (1975).

[4] D.T. Son, Phys. Rev. D59, 094019 (1999); T. Schäfer and F. Wilczek, ibid D60, 114033 (1999); D.K. Hong, V.A. Miransky, I.A. Shovkovy, and L.C.R. Wijewardhana, ibid D61, 056001 (2000), err. D62, 059903 (2000).

[5] R.D. Pisarski and D.H. Rischke, Phys. Rev. D60, 094013 (1999); D61 051501 (2000); D61 074017 (2000).

[6] M. Alford, K. Rajagopal, and F. Wilczek, Phys. Lett. B 422, 247 (1998).

[7] R. Rapp, T. Schäfer, E.V. Shuryak, M. Velkovsky, Phys. Rev. Lett. 81, 53 (1998); Annals Phys. 280, 35 (2000).

[8] J. Berges and K. Rajagopal, Nucl. Phys. B 538, 215 (1999); G.W. Carter and D. Diakonov, Phys. Rev. D60, 016004 (1999); T.M. Schwarz, S.P. Klevansky, and G. Papp, Phys. Rev. C60, 055205 (1999).

[9] D. Bailin and A. Love, Phys. Rep. 107, 325 (1984).

[10] D. Rischke, Phys. Rev. D62, 034007 (2000); G.W. Carter and D. Diakonov, Nucl.Phys. B 582, 571 (2000).

[11] A.J. Leggett, Rev. Mod. Phys. 47, 331 (1975).

12] T. Schäfer, Phys. Rev. D62, 094007 (2000).

[13] M. Alford, J. Bowers, and K. Rajagopal, Phys. Rev. D63, 074016 (2001); R. Rapp, E. Shuryak, and I. Zahed, Phys. Rev. D63, 034008 (2001).

[14] M.G. Alford, J.A. Bowers, J.M. Cheyne, and G.A. Cowan, hep-ph/0210106

[15] A. Schmitt, Q. Wang, D.H. Rischke, nucl-th/0301090

[16] A. Schmitt, Q. Wang, and D.H. Rischke, Phys. Rev. D66, 114010 (2002).

[17] J.A. Bowers, J. Kundu, K. Rajagopal, and E. Shuster, Phys. Rev. D64, 014024 (2001).

[18] A.L. Fetter and J.D. Walecka, Quantum theory of manyparticle systems, Mc Graw-Hill, New York (1971).

[19] G.W. Carter and S. Reddy, Phys. Rev. D62, 103002 (2000); P. Jaikumar, M. Prakash, T. Schäfer, astro-ph/0203088 I.A. Shovkovy, P.J. Ellis, hep-ph/0204132

[20] M. Alford and K. Rajagopal, hep-ph/0204001

[21] H. Nielsen and S. Chadha, Nucl. Phys. B105, 445 (1976); H. Leutwyler, Phys. Rev. D49, 3033 (1994); T. Schäfer, D.T. Son, M.A. Stephanov, D. Toublan, and J.J. Verbaarschot, Phys. Lett. B 522, 67 (2001); F. Sannino and W. Schäfer, Phys. Lett. B 527, 142 (2002).

[22] T.-L. Ho, Phys. Rev. Lett. 81, 742 (1998); T. Ohmi and K. Machida, J. Phys. Soc. Jpn. 67, 1822 (1998).

[23] V.A. Miransky and I.A. Shovkovy, Phys. Rev. Lett. 88, 111601 (2002).

[24] Strictly, $c_{v}=\left.(T / V)(\partial S / \partial T)\right|_{V, N}$, but the correction 
term is small [18]. 